\title{
A Comparative Study of Map Use
}

Niels Olof Bouvin

Christina Brodersen

Susanne Bødker

Allan Hansen

Clemens Nylandsted Klokmose

Department of Computer Science,

University of Aarhus

Denmark

n.o.bouvin@daimi.au.dk

sorsha@daimi.au.dk

bodker@daimi.au.dk

fah@daimi.au.dk

clemens@daimi.au.dk

\begin{abstract}
We present a study comparing the handling of three kinds of maps, each on a physical device: a paper map, a tablet-PC based map, and a cellular phone based one. Six groups of users were asked to locate eight landmarks, looking out a window, and using a particular map. We have begun analyzing video recordings of the situations, and this paper will give examples of the handling of the three kinds of physical devices.
\end{abstract}

Keywords: Map interaction, physical devices

ACM Classification Keywords: H5.2 User Interfaces

\section{The study rational and focus}

In an earlier project [3] kids were sent out to do project work in the streets. Some were equipped with annotated maps on a tablet-PC and others with cellphones. We videotaped this interaction only to realize the difficulty of capturing the detailed handling of the artifacts while the kids were moving about. To better compare map use and handling of the different physical devices we decided to set up the study outlined here.

\section{The three interaction technologies}

In the study, three kinds of maps were used on different devices: a paper map in a book, a digital map on a tablet-PC, and a digital map on a Nokia smart phone 
running the mobile hypermedia system HyConExplorer [3], developed at the University of Aarhus.

The technologies are characterised by different interaction techniques, but share some similarities: all three devices are relatively small and can be carried around, shared between users, and handed from one person to another while in use. The book format of the paper maps lets the users use their fingers to point to locations on the maps and to quickly "bookmark" related maps on other pages. The tablet-PC supports interaction with the maps through an electromagnetic pen with a single button. This allows users to use the pen much as an analogue pencil but at the same time select objects on the screen using the button like a computer mouse. The smart phone is a one-handed device controlled by a keyboard and a five-way joystick.

The three technologies support roughly the same set of navigation tools. The paper maps support panning from one map to another by a grid index printed at the margins of the pages. Viewing the same area at different scales is accomplished by turning pages. The scale of each map is printed in the corner of the map and can be used to manually measure distances. On the tabletPC, panning is supported by clicking on a point on the map. Different map scales can be displayed by invoking tools from a small palette, or by selecting a square area on the screen. The application has a dedicated tool for measuring distances between two points or along a series of segments. The HyConExplorer application on the smart phone uses a five-way joystick to control a small pointer on the screen. By clicking with the joystick, the map is panned and centred on the pointer position. Scaling is supported through menu-selectable scales. In the field, these tools can be automatically controlled through GPS [3]. Both the tablet-PC and smart phone retrieve maps from the net, which can cause a delay when panning and zooming.

\section{The experimental setup}

The study was performed with six groups with 2-3 members, all adults. Each group was given eight assignments-e.g., locating north, or identifying a local school and the distance and direction to it-half to be done with one technology, half with another. All assignments relate to objects that are (more or less) visible through a large window (as seen in Figure 2).

The experiments were recorded using the setup shown in Figure 1 plus a tripod-mounted digital camera. The top-mounted camera provided a general view of the participants' posture and gestures, and the table mounted camera provided a close-up of their interactions with the map technology at hand.

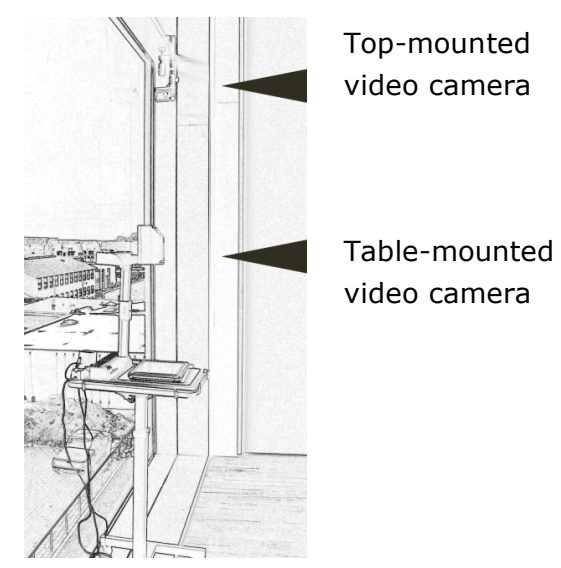

Figure 1: The Experimental Setup 


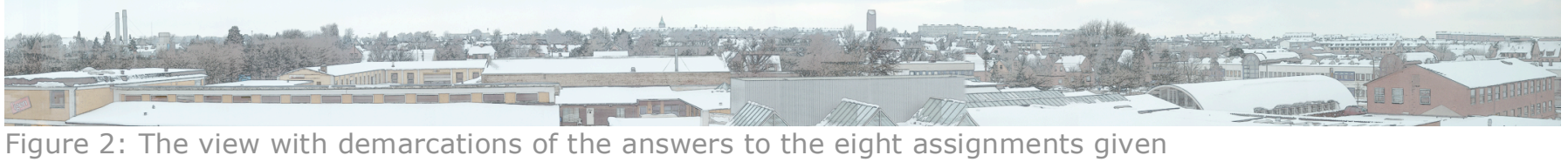

\section{The analysis}

The video material has been analyzed in three steps, in interaction with the theoretical model (Figure 3). First, we logged and transcribed the video, letting the material speak in the manner of interaction analysis [4]. Second, the model below was developed, inspired by activity theory [1], [2] and rooted in the empirical analysis. In the third step, we gathered incidents and compared these across technologies. We have primarily used a qualitative approach, despite the rigid set-up.

\section{Theoretical model}

All assignments result in two people agreeing on a target, common orientation, distance, etc. (the answer). They possess some knowledge about the landscape that they are looking at and the technology that they are applying (their orienting basis). They create a common orienting basis, mediated by the individual orienting bases as well as by the maps and technologies applied and the view through the window. Based on the assignment, the individual and common orienting bases, and the maps/technology, the groups perform a number of tasks to establish the outcome. These tasks are neither isolated from each other, nor carried out in one particular order. However, the assignments give a main direction of the tasks leading from $A$ to $F$. Iteration is frequent. Each task is mediated by the map, the particular device, the outside view, and the orienting bases of group members, as we shall illustrate.

\section{Carrying out the analysis}

Activity-theoretical HCI provides a number of techniques for carrying out an analysis of use. We used checklists [2] to understand the context of the events studied, after which we sampled instances of how each task was carried out, and decomposed them further [1]. The tasks were categorized, and further incidents gathered. The orienting basis was traced through speech and bodily references. Fundamental to our understanding of the role of the three technologies are the breakdowns that happen.

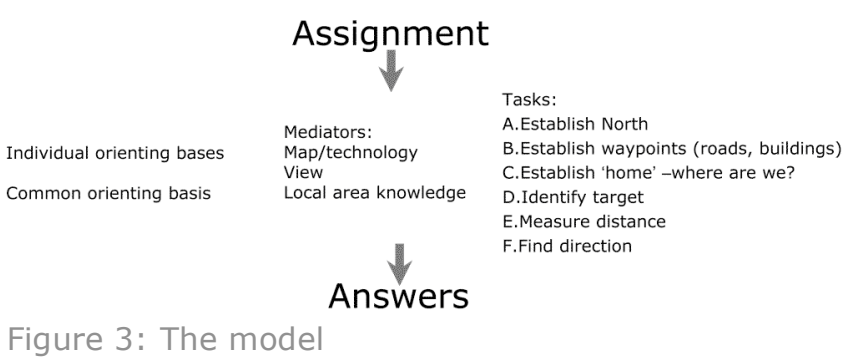

\section{Examples}

In the following, we will give preliminary examples of the analyses that we are currently carrying out, showing the richness of our material. 
Visible identification of target

This task results in the creation of the initial common orienting basis for the group, with respect to the particular assignment. We see in the video two overall strategies with sub-strategies: $\mathrm{A}$. Both $(\mathrm{A} 0)$, or one group member can make a visual identification of the target and point it out to the other (A1), or negotiate the location through scanning the landscape, talking out loud and pointing (A2); and B. none of the group members can make an identification of the target and they go through iterations of looking at the map (B1), looking out (B2), and trying to remember from past experience (orienting bases) (B3) until they identify the target and end the iteration in one of the three substrategies. Furthermore, there is a strategy $C$ which is basically a failure to identify the target.

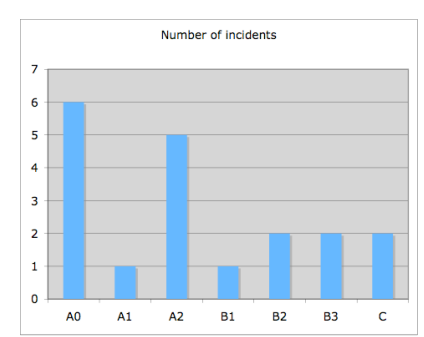

Figure 4: Distribution of incidents

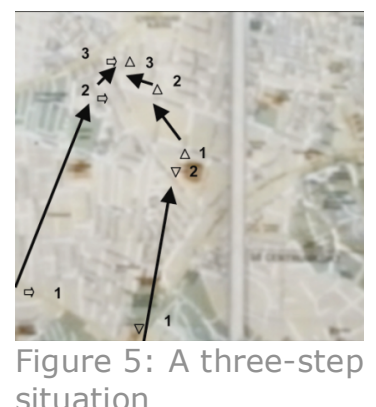

situation
When counting the number of incidents (Figure 4) in each category, we see that only 1 out of 19 incidents leads to the target being identified on the map (B1), in 2 out of 19 (C) there is a failure to identify the target, and in the rest the target is found because the target is known to the group or could easily be identified visibly ("the yellow buildings at a distance"). One assignment focuses on a target that is not easily seen (6-the local school). The B1 group makes the identification by looking at the paper map which shows names of schools and other public buildings. The two groups, who fail (C), do so because neither the cell-phone (group 1) nor the tablet-PC (group 2) gives such support. Several other assignments could have been solved using paper maps in much the same way. Nonetheless, people tend to make a visual identification first. In the future research we will go into further detail with this type of analysis and we aim to get a better understanding of the relationship between what is known by group members, what can be seen, and the maps.

Using the map to make detailed identification The next step in the procedure is from visual identification to identification on the map. We identified, based on the transcript, the elements of the procedures and how they were interconnected. We were particularly concerned with procedures carried out using (or not) the cell-phone and the tablet-PC, respectively.

Two kinds of procedures stand out: A. identification through landmarks, and B. grid-based identification of roads. All participants seem to have a dual purpose of creating a common orienting basis, and of being able to point at something outside, or on the map.

For the cell-phone, we have the four main examples, which each include several instances of both types of navigation: 5 instances of grid-based mapping and 6 landmarks. Landmarks are anchor points in the common orienting basis. In the following example, the group looks only at the map, using the common orienting basis to place the building on the map: 


A: This is Skovvejen where I live, and then it is further up Rand-
ersvej
B: but, it is on the corner of Nordre Ringgade, so it is right there//
A:// Right where we are
B: So//

Regarding grid-based identification, it is characteristic of the instances we have sampled that the superimposing of the map and street names upon what can be seen is very local and rather depending on people's previous knowledge of the terrain. When discussing which road is which, our users tended to rely on their knowledge of the environment:

E: Then perhaps it is up there
D: Vågøgade
C: No it is the one that passes, passes, at it comes from down
Defta. What is it called?
D: There is a road without a name
C: What is it called? It is called...

These analyses will be supplemented with an analysis of the use of the tablet-PC, in order to focus on the ways in which these technologies support navigation strategies.

\section{Handling the maps}

It is extremely common that both persons rest both hands on the paper map, or hold one or more fingers on particular locations. An example from one group serves to illustrate this: One of the participants (A) rests his right hand on the paper map while identifying where the group is (home) with the right hand. He maintains this position of the hands while looking out the window. The group makes visual identification of the target, and their gazes move back to the map. At the same time, A starts to move his right hand towards the location of the target, while $B$ places his right hand near the target, and $A$ moves his left hand towards home (figure 5 and $6 \mathrm{a}$ ). In the third step, A and B both point at the target with their right hands.

People do not rest their hands on the tablet-PC. In very few instances a hand or the pen is held over the tablet to e.g., indicate a direction, or the pen and/or finger is used to point on the map (figure $6 \mathrm{~b}$ ).

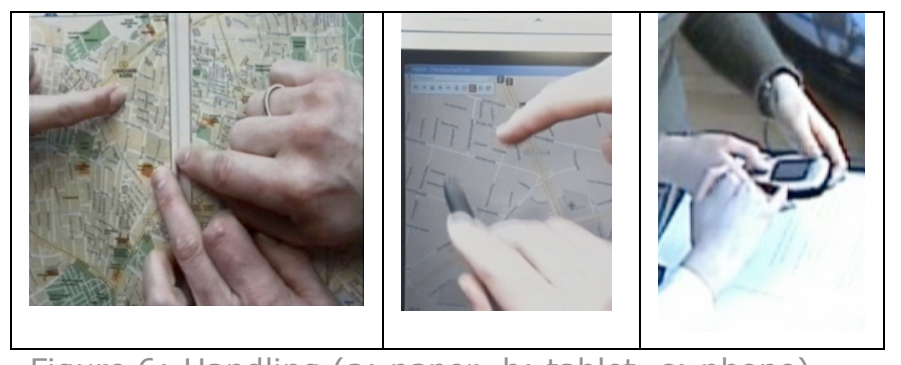

Figure 6: Handling (a: paper, b: tablet, c: phone)

There are several, shorter examples where both users have their hands on the phone, e.g., one person holds the phone and both point (figure $6 \mathrm{c}$ ). In at least one example, a person tries to activate the joystick while another person is holding. In several instances, the phone gets handed over from one person to another as part of the unfolding action. Holding the phone and resting on the paper map serve some of the same purposes, as a kind of homing. We will analyze this in further detail with particular focus on the lack of hands-on handling of the tablet.

\section{Finding north and other directions}

Throughout the assignments, the participants were asked to identify the direction for their target. Specifically, assignment 1 was an identification of the direc- 
tion 'north' which we hoped would serve as a good starting point for aligning the view through the window with the view provided by the particular map. We were also curious as to how the different technologies could support their subsequent negotiation about direction, if at all (tasks $A$ and $F$ in the model). Preliminary analysis strongly suggests that the success of identifying a direction is based on the success of the teams to connect their visual identification of the direction with the identification of the same direction on the map. This is achieved through choosing a visual landmark or a target through the window and comparing this to similar waypoints and/or the knowledge about the map's orientation. More detailed analysis will determine what role the technology plays in this context.

\section{Conclusion and lessons for redesign}

We have developed a first theoretical model of the participants' use of the different maps and technologies. The participants solved most assignments by first creating a common orienting basis in the model. The common orienting basis is mediated by the individual orienting basis of each person in the group (prior experience and knowledge), the employed technology, the map, and the view (the physical world).

We saw different procedures for going from a visual identification of a target to the identification on the map. Two procedures for aligning the map with the physical view stood out: landmarks and grid-based identification. We will analyze and develop these further: e.g., how we may place waypoints on the electronic maps and what would be the effect of using them; and how we may support the grid-based procedure through design.
The assignments and set-up gave least justice to the cell-phone, because it has its main force when on the move in the field. However, the study gave a substantial amount of immediate feedback to the design of, in particular, our own HyConExplorer.

The paper map affords homing with the hands on the map that neither of the other technologies do. This seems important for both applying local area knowledge in relation to map information, and for relating map and landscape. Especially, when none of the group members could make an immediate identification of a target, homing was used a quick way to iterate between looking at the map, looking at the world, and discussing the correspondence between the two based on the individual's past knowledge and the common orienting basis. The question is then: how can homing be supported on the electronic maps to support these activities?

\section{References}

[1] Bedny, G. Z., Seglin, M. H., \& Meister, D. (2000). Activity theory: history, research and application. Theoretical Issues in Ergonomics Science, 1(2), 168-206.

[2] Bertelsen, O. \& Bødker, S. Activity theory (2002). HCI Models, Theories and Frameworks (ed. John Carroll). Morgan Kaufmann, pp. 291-324.

[3] Bouvin, N. O, Brodersen, C., Hansen, F. A., Sejer, O. I., and Nørregaard, P. 2005: Tools of Contextualization: Extending the Classroom to the Field. In Proc. of the $4^{\text {th }}$ Int. Conf. for Interaction Design and Children (IDC 2005), June 2005, Boulder, CO, USA.

[4] Trigg, R., Bødker, S. \& Grønbæk, K. (1991). A Video-based Analysis of the Cooperative Prototyping Process. Scandinavian Journal of Information Systems, vol. 3, pp. 63-86. 